This item was submitted to Loughborough's Research Repository by the author.

Items in Figshare are protected by copyright, with all rights reserved, unless otherwise indicated.

\title{
Crossing minimisation Heuristics for 2-page drawings
}

PLEASE CITE THE PUBLISHED VERSION

PUBLISHER

(C) Elsevier

LICENCE

CC BY-NC-ND 4.0

\section{REPOSITORY RECORD}

He, Hongmei, Ondrej Sykora, and Imrich Vrt'o. 2019. "Crossing Minimisation Heuristics for 2-page Drawings". figshare. https://hdl.handle.net/2134/2380. 
This item was submitted to Loughborough's Institutional Repository by the author and is made available under the following Creative Commons Licence conditions.

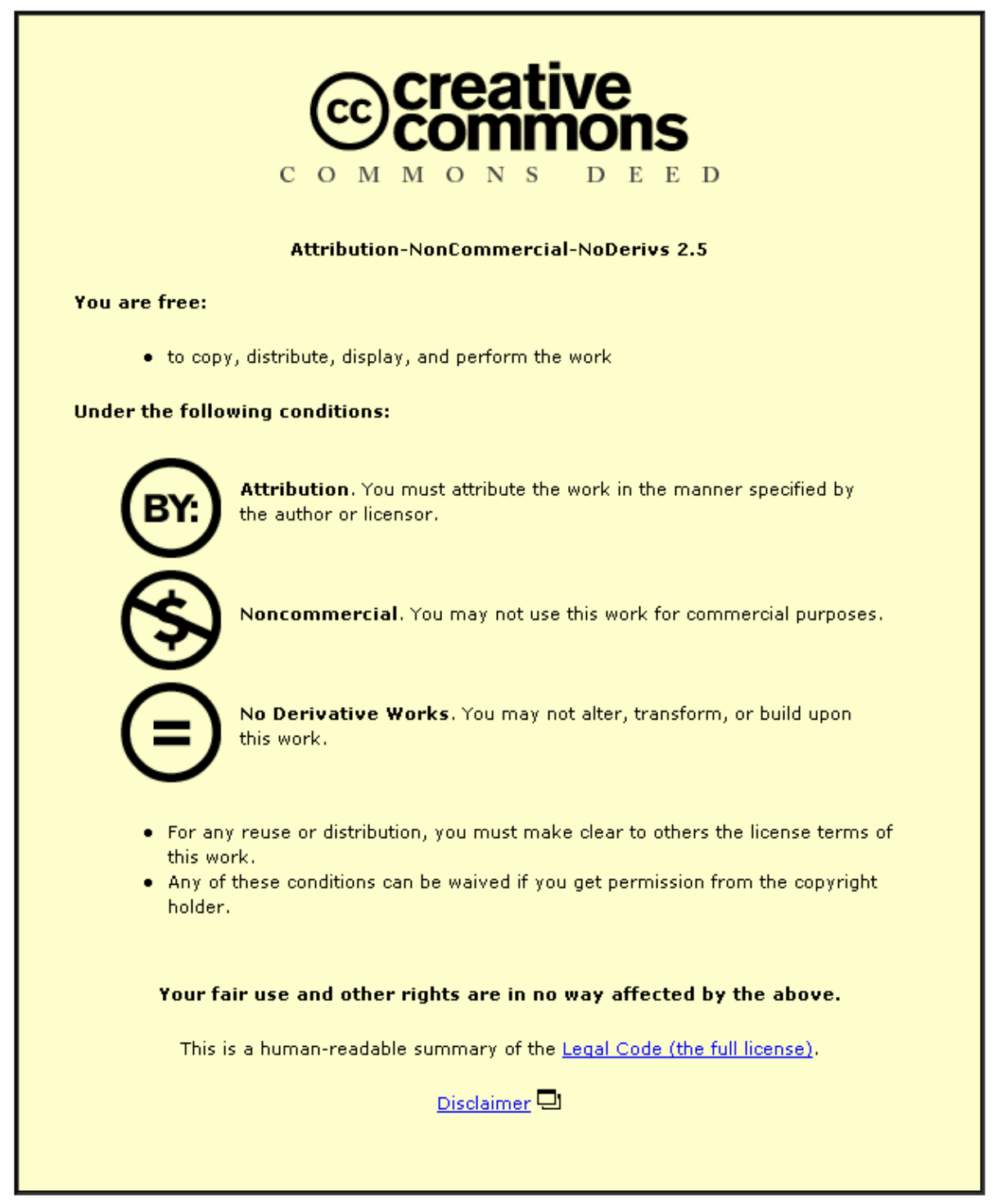

For the full text of this licence, please go to: http://creativecommons.org/licenses/by-nc-nd/2.5/ 


\title{
Crossing Minimisation Heuristics for 2-page Drawings
}

\author{
Hongmei He, Ondrej Sýkora ${ }^{1,2}$ \\ Department of Computer Science \\ Loughborough University \\ Loughborough, Leicestershire LE11 3TU, the United Kingdom \\ Imrich Vrt'o ${ }^{3}$ \\ Department of Informatics \\ Institute of Mathematics, Slovak Academy of Sciences \\ Dúbravská 9, 842 35 Bratislava, Slovak Republic
}

\begin{abstract}
The minimisation of edge crossings in a book drawing of a graph $G$ is one of important goals for a linear VLSI design, and the two-page crossing number of a graph $G$ provides an upper bound for the standard planar crossing number. We propose several new heuristics for the 2-page drawing problem and test them on benchmark test sets like Rome graphs, Random Connected Graphs and some typical graphs. We get exact results of some structural graphs, and compare some of the experimental results with the one in paper[2].
\end{abstract}

Keywords: 1-page crossing number, 2-page crossing number, 2-page drawing, book crossing number, Hamiltonian cycle 


\section{Introduction}

The simplest graph drawing method is that we put vertices of a graph on a line and draw the edges above the line. Such a drawing corresponds to the linear VLSI design and edge crossing minimisation is one of important goals in such a design, since smaller number of crossings means cheaper design. The edges can be put in one or more half-planes (pages). Similarity to a book: vertices on a line (backbone), and edges in half-planes (pages) gave the name to this drawing: book drawing. The minimal possible number of edge crossings is called book crossing number. One-page [10] crossing number corresponds to outerplanar [4] (also called convex [11], or circular [12]) crossing number, which is the minimal possible number of pairs of crossing edges in a drawing where one places vertices of a $n$-vertex $m$-edge connected graph $G=(V, E)$ along a circle, and the edges are drawn as straight lines. Therefore one-page drawing can be reduced to the task to find an ordering $f: \mathrm{V} \rightarrow\{0,1, \ldots n$ $1\}$ of the vertices, minimising the number of edge crossings. We denote onepage crossing number as $v_{1}(G)$, following the notation in [10]. The problem has been proved to be NP-hard problem [5]. The 2-page drawing can be simplified similarly: one places vertices of an $n$-vertex $m$-edge connected graph $G=(V, E)$ along a circle called and every edge is completely drawn in one of two colours. The two-page crossing number of a graph $G$ is the smallest possible number of crossings of edges of the same colour, and we denote it as $v_{2}(G)$. The problem is also NP-hard [6]. Given the two ends of an edge, $i$ and $j$, and the two ends of the other edge, $k$ and $l$, in the current layout, if $i<k<j<l$, then the two edges of same colour will produce a crossing. So if we define each edge in adjacency matrix with the number of the page where the edge is on, we can calculate the number of crossings in a $\kappa$-page drawing of a graph $G$ with the following formula:

$$
v_{\kappa}(G)=\sum_{i=0}^{n-4} \sum_{j=i+2}^{n-2} \sum_{k=i+1}^{j} \sum_{l=j+1}^{n-1} \operatorname{adj}[i, j] \bigodot \operatorname{adj}[k, l]
$$

where

$$
\operatorname{adj}[i, j] \bigodot \operatorname{adj}[k, l]=\left\{\begin{array}{l}
1 \text { if } \operatorname{adj}[i][k]=\operatorname{adj}[j][l] \neq 0 \\
0 \text { if otherwise }
\end{array}\right.
$$

\footnotetext{
1 This research was supported by the EPSRC grant GR/S76694/01 and by VEGA grant No. 2/3164/23

2 Email: H.He@lboro.ac.uk

3 Email: Imrich.Vrto@savba.sk
} 
The main motivation for studying one and two-page crossing number problem is the fact, that they provides an upper bound for the standard planar crossing number and seems to be easier.

Recently, a lot of heuristic algorithms were designed for one-page drawing problem, e.g. algorithm of Mäkinen [7], CIRCULAR algorithm [12], algorithm of Baur and Brandes [1], and AVSDF [3]. The 2-page crossing number as an approximation to the plane crossing number was first studied in $[8,9]$ but no thorough testing was done there. The most important paper in this area is [2], where first an order of vertices for some structural graphs is given by a hamiltonian cycle, and then eight different heuristic algorithms to find good distribution of edges between two pages were designed and tested. Recently, Winterbach [13] proposed heuristics for the 2-page crossing numbers and applied them to estimating the plane crossing number of some small complete multipartite graphs.

In this paper we design new 2-page drawing heuristic algorithms based on the latest 1-page drawing algorithms [1] and [3], and compare different edge distribution strategies. We compare some of our results with [2] and the theoretical results published in [10].

For our experiments we used benchmark test suites:

Random Connected Graphs (RCGs) with different densities and sizes, which were used in paper[3].

Rome Graphs, which are taken from the test suite of GDToolkit and were utilized in $[1,3]$. There are two subsets of undirected graphs.

-RND_BUP: this graph set contains about 200 graphs generated randomly. Each graph in the set is biconnected, undirected and planar.

-ALF_CU: this graph set contains about 10,000 connected and undirected graphs

Special Graphs: including random 3-regular graphs, Halin graphs, meshes, complete p-partite graphs, complete graphs, Circulant graphs, hypercubes, and Cartesian Graphs.

\section{One-page drawing algorithms}

\subsection{Algorithm of Baur and Brandes}

The best algorithm of Baur and Brandes [1] consists of two phases: greedy and sifting.

Greedy phase: at each step a vertex with the largest number of already placed neighbours is selected, where ties are broken in favour of vertices with 
fewer unplaced neighbours, and then appended to the end that yields fewer crossings of edges being closed with open edges. The running time is $O((n+$ $m) \log n)$.

Sifting phase: Every vertex is moved along a fixed ordering of all other vertices. The vertex is then placed in its (locally) optimal position. The phase can be run in $O(\mathrm{~nm})$ time.

We use $\mathrm{BB}$ to denote the greedy phase, and $\mathrm{BB}+$ to denote the combination of two phases.

\subsection{AVSDF+ algorithm}

AVSDF + algorithm consists of two phases too: greedy and adjusting.

\section{Greedy Algorithm:}

AVSDF [3] is a variation of the DFS algorithm. At first one places the vertex with the smallest degree, and then visits the adjacencies of the current vertex, which have not been visited yet, such that the smallest degree vertex has the highest priority for visiting. The running time of the AVSDF algorithm is $O(m)$.

\section{Postprocessing phase(adjusting) [3]}

In this phase a vertex with the largest number of crossings caused by its incident edges is selected first, and then the best position among the current one and the ones next to its adjacent vertices is found. The procedure is repeated until no vertex can be adjusted. The running time is $O(m n)$. We denote AVSDF plus adjusting as AVSDF+.

\section{Two-page drawing algorithms}

\subsection{Slope strategy}

Edges of a graph are distributed on one of two pages according to their slopes. If the angle between an edge and the horizontal axis is larger than $90^{\circ}$, the edge is put on page 2, otherwise the edge is put on page 1. According to the position of vertices incident to the edge, we get the rough relationship between the slope value and positions of two ends of the edge, if $(i+j<|V| / 2$ or $i+j>=|V|$ and $i+j<3 \times|V| / 2)$ the angle between the edge and the horizontal axis is larger than $90^{\circ}$. We denote it as "SLOPE". The running time is $O(m)$. 


\subsection{Hybrid of one-page algorithm and edge pre-assignment:}

This is a hybrid heuristic algorithm of AVSDF and edge distribution, which places an edge incident to the currently placed vertex based on the smallest crossings produced by the edge and already placed edges. We denote it as "AVSDF_EP".

\subsection{Place edges according to their length (this is a strategy taken from [2])}

The longer length of edge is, the larger is the probability of its crossing with other edges. Whether the edges with length $=1$ are on page 1 or page 2 , they do not create any crossing. Therefore we create a sorted edge list without the edges with length $=1$, on non-increasing length, and then place each edge in the list in turn. The process is iterated until there is no improvement or iterations were done 5 times (the number 5 was found to be sufficient experimentally). We denote it as "LEN". The running time is $O(m)$.

\subsection{Adjust edges according to descending crossings}

The strategy is based on an initial one-page drawing. We start the edge which creates most crossings and put it to the other page, then recalculate the crossings created by each edge, sort edges according to the number of crossings created by each edge, and repeat the process until no edge can be adjusted. We denote it as "CRS". The worst case running time is $O\left(\mathrm{~m}^{2}\right)$ (the best case running time is $O(m)$ ).

\section{Experiments}

The experiments in paper [2] were done based on a fixed Hamiltonian cycle for some special structural graphs. Unfortunately, not every Hamiltonian cycle corresponds to an optimal vertex ordering, and an optimal drawing might not correspond to a Hamiltonian cycle. Moreover, for an arbitrary graph, a Hamiltonian cycle might not exist, or even if it exists, we might not be able to find it. Our experiments aim at finding the relationship between one-page drawing and two-page drawing, and what is the most important factor to affect the crossings. So experiments are done on a variety of graphs described previously from the following aspects. 


\subsection{Test of two page drawing algorithms}

We compare pairs of algorithm combinations formed by 2 heuristics for onepage drawing and 4 strategies of edge distribution, and explore the relationship between one-page and two-page drawings on ALF_CU, RND_BUP, RCGs, and 3-regular graphs.

\subsection{Test on RCGs with different edge density}

We compare the combinations, AVSDF+_LEN, BB+_LEN and AVSDF_CRS and $\mathrm{BB}+$ _CRS on RCGs with edge density $1 \%, 2 \%$, and $5 \%$. Furthermore we explore the affect of density.

\subsection{Typical graph test}

For some typical classes of graphs as complete p-partite graphs, Halin graphs, 3 -row meshes, $m_{3 \times n}$, we know optimal one-page drawings, or for some other graphs as 4-row meshes, $m_{4 \times n}$, hypercubes, we have a hypothesis of optimal one-page drawings, so we can first find optimal one-page drawings and then apply a strategy of edge distribution. This method of experiments was used by [2] too. We test some Circulant graphs used in [2], but we first apply a heuristic algorithm to find a good one-page drawing, and then apply a strategy of edge distribution. In most cases, we get similar results, but for some Circulant graphs as C38(1,7), C40(1,5), and C42(1,4), we get some interesting results, which are even better than the optimal values listed in [2]. We have the exact results: $\nu_{2}\left(m_{3 \times n}\right)=0 ; \nu_{2}\left(m_{4 \times n}\right)=0$; and $\nu_{2}($ Halin $)=0$, and present solutions for them.

\section{Conclusion}

From our experiments, one-page drawing as a base of two-page drawing is very important, but it is not absolute. Two-page crossing number is not only related to the one-page drawing, but also related to the edge distribution and the structure of graphs. For Rome graphs, "AVSDF_CRS" and "BB_CRS" get best results; For RCGs, "BB_CRS" gets best results; For Halin graphs and Cartesian graphs, $C m \times C n$, "AVSDF_CRS" gets best results; For Circulant graphs, $C_{k n}(1, n)$, "BB_LEN" and "BB_CRS" get best results; Based on a fixed optimal order of vertices, for Circulant graph, "LEN" and "CRS" get best results, and for complete graphs and complete p-partite graph, "SLOPE" gets best results. The hybrid algorithm, "AVSDF_EP", sometime gets bet- 
ter results, but almost all of results by "AVSDF_EP" can't be improved by a following other strategy of edge distribution, this could be because that "AVSDF_EP" gets a local optimal solution, and the vertex order and edge distribution are in a kind of "deadlock". "SLOPE" strategy stays in the shade of the "LEN" strategy and "CRS" strategy for most graphs. However, there is a good use of "SLOPE" as a preprocessing of edge distribution after a good vertex order is provided. In summary, a good one-page drawing+"SLOPE" + "CRS" is the best combination in our experiments. With the rise of edge density, the results by "LEN" is closer to the ones by "CRS". We present some exact results for Halin graphs, p-partite complete graphs, 3-row meshes, 4-row meshes and etc. Moreover with our 2-page strategies based on a 1-page algorithm, for some Circulant graphs, we get interesting results, which are even better than optimal values listed in [2]. We will pay attention to the study of Cartesian graphs, $\mathrm{Cm} \times \mathrm{Cn}$ in our further work.

\section{References}

[1] Baur, M., Brandes, U., Crossing Reduction in Circular Layouts, in: Proc. 30th Intl. Workshop Graph-Theoretic Concepts in Computer-Science (WG '04), LNCS 3353, Springer, Berlin, 2004, 332-343.

[2] Cimikowski, Robert: Algorithms for the fixed linear crossing number problem, Discrete Applied Mathematics 122 (2002), 93-115.

[3] He, H., Sýkora, O, New circular drawing algorihtms, in: Proc. ITAT'2004.

[4] Kainen, P.C., The book thickness of a graph II, Congressus Numerantium 71 (1990), 121-132.

[5] Masuda, S., Kashiwabara, T., Nakajima, K., Fujisawa, T., On the NPcompleteness of a computer network layout problem, in: Proc. IEEE Intl. Symposium on Circuits and Systems 1987, IEEE Computer Society Press, Los Alamitos, 1987, 292-295.

[6] Masuda, S., Kashiwabara, T., Nakajima, K., Fujisawa, T., Crossing minimization in linear embeddings of graphs, IEEE Trans. Comput. 39 (1990) $124-127$.

[7] Mäkinen, E., On circular layouts, International Journal of Computer Mathematics 24 (1988), 29-37.

[8] Melikov, A.N., Koreičik, V.M., Tiščenko, V.A., Minimization of the number of intersections of edges of a graph (Russian), Vyčisl. Sistemy Vyp. 47 (1971), $32-40$. 
[9] Nicholson, T.A.J., Permutation procedure for minimazing the number of crossings in a network, Proc. Inst. Elec. Engnrs. 115 (1968), 21-26.

[10] Shahrokhi, F., Sýkora, O., Székely, L.A., Vrt'o, I.: The book crossing number of a graph, Journal of Graph Theory 21 (1996), 413-424.

[11] Shahrokhi, F., Sýkora, O., Székely, L.A., Vrt'o, I., Towards the theory of convex crossing numbers, in: Towards a Theory of Geometric Graphs, ed. J. Pach, Contemporary Mathematics, Vol. 342, American Mathematical Society, Providence 2003, 249-258.

[12] Six, J.M., Tollis, I.G., Circular drawings of biconnected graphs, in: ALENEX'99, LNCS 1619, Springer, Berlin, 1999, 57-73.

[13] Winterbach, W., The crossing number of a graph in the plane, Master Thesis, Dept. Appl. Math., University of Stellenbosch, SA, 2005, 334 p. 